\title{
De Wittenberg à Wittenberg : le thème du retour dans Dr. Faustus
}

\section{Claude Peltrault}

\section{OpenEdition}

\section{Journals}

Édition électronique

URL : http://journals.openedition.org/shakespeare/515

DOI : 10.4000/shakespeare.515

ISSN : 2271-6424

\section{Éditeur}

Société Française Shakespeare

\section{Édition imprimée}

Date de publication : 1 novembre 1989

Pagination : 67-80

\section{Référence électronique}

Claude Peltrault, "De Wittenberg à Wittenberg : le thème du retour dans Dr. Faustus », Actes des congrès de la Société française Shakespeare [En ligne], 6| 1989, mis en ligne le 01 janvier 2007, consulté le 26 avril 2019. URL : http://journals.openedition.org/shakespeare/515 ; DOI : 10.4000/ shakespeare. 515 


\section{SOCIETE FRANCAISE SHAKESPEARE}

\section{Actes du Congrès - 1984}

\section{LIEU ET TEMPS}

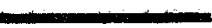

Directeur de la publication Jean FUZIER 


\section{De Wittenberg à Wittenberg : le Thème du Retour dans Doctor Faustus}

Suivant que le respect l'emporte ou non sur la perplexité, la critique a pour habitude de considérer le Doctor Faustus de Marlowe comme une pièce ambiguë ou incohérente, en tout cas dépourvue d'unité, surtout à partir de la scène viii. Par la magie, Faust parcourt tous les lieux imaginables et fait revenir le temps passé dans le présent, les morts chez les vivants, lui même passe pour un revenant lorsqu'il sème la perturbation au palais pontifical. C'est précisément ce thème du retour, pris avec ses connotations spatiales, temporelles et religieuses, que je propose comme fil conducteur dans la recherche de l'unité de la pièce, en m'en tenant au texte de $1616^{1}$.

Rappelons d'abord que le temps peut être considéré à la fois comme le processus linéaire fini qui, dans une perspective matérialiste, conduit l'être vivant de la naissance à la mort, et comme une suite d'oscillations entre deux pôles. Ce va-et-vient nous renvoie aux origines mythiques du temps : l'union du ciel et de la terre, à Cronos qui avale et vomit ses enfants, aux allées et venues de Perséphone 
entre le monde d'en haut et le monde d'en bas, à la circulation cyclique des âmes selon Platon etc... Le mythe de l'éternel retour ${ }^{2}$ répond à l'angoisse des besoins matériels devant la terre morte en apparence, mais que l'on sait seulement endormie et grosse de la moisson prochaine. Il répond aussi à l'angoisse métaphysique devant le néant, la mort devient comme la nuit du jour, la tombe comme un ventre d'où l'on renaît. ${ }^{3}$ Le christianisme fonde l'assurance d'une vie nouvelle et éternelle après la mort, sur la résurrection du Christ. Saint Augustin, méfiant envers ce qu'il est convenu d'appeler les "survivances païennes", prend bien soin de distinguer l'idéologie chrétienne du mythe pré-chrétien, dans La Cité de Dieu: [cette éternité] "ne sera pas l'alternance continue du départ des uns par la mort, de l'arrivée des autres par la naissance, qui fera paraître en elle un semblant de perpétuité, comme dans un arbre revêtu d'une frondaison continue, la même verdure se maintient quand les feuilles qui sèchent et tombent sont aussitôt remplacées par d'autres qui naissent [...], mais en elle tous les citoyens seront immortels, car les hommes aussi obtiendront ce que n'ont jamais perdu les saints anges. ${ }^{4}$ Tout en niant l'alternance, il décrit la vie éternelle comme le retour à un état ancien, perdu par la faute de l'homme, et regagné par le dieu fait homme, pour l'éternité. Ce n'est pas "l'éternel retour", mais "le retour éternel ", les fêtes liturgiques vont être célébrées selon un rythme cyclique parallèlement aux ancestrales coutumes saisonnières, et tout cela, ponctué par le branle des cloches, s'intègre sans difficulté dans le schéma familier du mythe du temps.

A la lumière de ces définitions, faisons rapidement l'inventaire des références spatio-temporelles les plus évidentes dans DF. Le mythe d'Icare traduit en termes de spatialisation le châtiment d'une ambition conçue comme transgression, et la première image scénique nous montre un intellectuel désabusé, (dé)-fini par un espace clos : "And this the man that in his study sits" (Prologue, 28). Puis, Faust passe en revue les domaines du savoir comme autant d'entités linéaires parcourues de $\mathrm{A}$ à $Z,{ }^{5}$ dont les limites entravent ses aspirations, mais en même temps, il ne peut imaginer le destin humain que comme enfermé dans l'espace verbal du syllogisme, logique mortelle qui verrouille les issues de secours vers le salut. Il se tourne alors vers la magie qui semble seule permettre l'élan vers les espaces infinis et la renommée éternelle, substitut de la vie éternelle. Le 
thème $d u$ dépassement entre très vite en contradiction avec deux principes qui régissent sa pensée et son comportement: celui de l'enfermement et celui du retour. ${ }^{6} \mathrm{Ce}$ " forward wit " (Epilogue, 5), ira en régressant par rapport à ses nobles aspirations, et ce qui ravit l'apprenti magicien qui rêve d'enfermer l'Allemagne dans un cylindre de cuivre, de détourner le cours du Rhin pour encercler Wittenberg, ce sont, justement, le cercle dont il s'entoure pour invoquer les démons, les livres où sont enfermés les secrets, les mots que l'on peut retourner à l'envie "backward and forward", ou la folle ronde des lettres dans les anagrammes. Son premier acte de magicien consiste à vérifier le fonctionnement d'un système de va-et-vient entre ce monde et l'enfer dont Mephostopheles ${ }^{7}$ sera le go-between : "I charge thee to return and change thy shape ; /... Go and return and old Franciscan friar" (iii, 25 et 27), système parodié dans la scène iv avec le calembour grivois sur les "comings-in" et les "goings-out" de Robin (iv, 5 et 6). Lui-même brûle d'expérimenter le système : « $O$, might I see Hell and return again safe, how happy were I then!" (vi, 172). Dans le monologue à Hélène, cette obsession du va-et-vient superpose aux connotations blasphématoires du "Heaven is in those lips » (xviii, 104), des connotations érotiques allant jusqu'à l'extase :

Her lips suck forth my soul : see where it flies!

Come, Helen, come, give me my soul again $(102-103)^{8}$.

Plus bas, Faust s’identifie, à travers Pâris, à la flèche, symbole du membre viril :

Yea, I will wound Achilles in the heel

And then return to Helen for a kiss (110-111).

Le rythme "coïtal " du passage renvoie directement au mythe sexuel de la création du temps évoqué plus haut, et nous allons voir qu'à ces connotations érotiques se mêlent des connotations religieuses.

Le retour à Wittenberg au terme du pacte confirme l'obsession de Faust, et ouvre la porte à une interprétation qui dépasse celle de Susan Snyder, lorsqu'elle compare, à juste titre, la vie du magicien à une vie de saint à l'envers. ${ }^{9}$ Notons d'abord cette étonnante précision 
qui ne figure pas dans la source connue de Marlowe : "I have been a student here these thirty years" (xix, 45), et souvenons-nous que Jésus, au terme de sa mission, revint aussi sur ses pas, vers Jérusalem, la ville où trente ans plus tôt il avait été présenté au temple. Or, qu'est-ce que Wittenberg sinon le temple du protestantisme depuis que Luther y a rendu publiques ses fameuses thèses? De plus, Jésus et Faust ont passé leur vie d'homme à enseigner et à faire des prodiges. La comparaison, déjà suggérée par le consummatum est de la scène du pacte, souligne cruellement, par l'antithèse, les limites de ce retour de Faust, car pour Jésus il préfigure la résurrection, le retour à la maison du Père, et le retour éternel offert à l'humanité. Pour le chrétien, l'acte de baptême authentifie sa filiation avec Dieu, et ses droits à ce que l'Église, dans le symbole de Nicée (en 325), appelle "l'héritage". Les théologiens protestants, et en particulier Calvin, insistent sur cette notion. Le baptême "nous est envoyé de [Dieu] comme une lettre patente signée et scellée " ${ }^{10}$ car "nous avons été adoptés en Christ du Père Céleste, pour être ses enfants et ses héritiers " (IRC, III, xv, 5, p. 270). Dans cette perspective on comprend mieux le légalisme tatillon de Mephostopheles, car le pacte avec le diable constitue un acte de baptême à rebours par lequel le signataire renie sa filiation et choisit Satan pour père. Non seulement il perd tous ses droits à l'héritage, c'est-à-dire au retour éternel à la maison du Père, mais c'est lui qui devient "body and soul, flesh, blood, or goods" (v, 110), propriété du diable à sa mort. Marlowe effleure très subtilement le sujet dès le monologue de la scène $i$, où Faust cite un passage du code de Justinien relatif à l'héritage :

Exhereditare filium non potest pater nisi-

Such is the subject of the Institute

And universal body of the law. (i, 31-33).

Il me semble que cet "Institute " renvoie indirectement à Calvin, et à son Institution de la Religion Chrétienne dont Marlowe avait eu tout le temps de se pénétrer à Cambridge. ${ }^{11}$ La signature du pacte, viol de la loi divine et violence au temps reconquis, s'accompagne, chez Marlowe, des mêmes connotations érotiques que le monologue à Hélène : le poignard $(\mathrm{v}, 49)$, le sang qui coule des lèvres de la plaie $(\mathrm{v}$, 54 et 57). Le bras, poignard poignardé, rappelle le mythe de la naissance de Cronos, venu au monde en châtrant son père Uranus avec 
une faux, inaugurant par le geste symbolique du faucheur le mouvement pendulaire du temps. La métaphore de l'épilogue: "Cut is the branch that might have grown full straight " (1), fait écho à "Stab thy arm courageously " $(\mathrm{v}, 49)$, l'acte qui brise l'élan de l'homme vers le retour éternel à Dieu, dont le gage est la résurrection, doit s'exprimer par la cassure d'un symbole d'érection. ${ }^{12}$

Dès lors, qu'en est-il de la durée humaine? Fidèle à sa source, Marlowe lie le diable à Faust pour vingt-quatre ans, mais le nombre se trouve fixé comme à l'étourdie, après l'emploi d'expressions vagues : "whilst I live " (iii, 38), ou " some certain day" $(\mathrm{v}, 50)$. Alors, pourquoi vingt-quatre ? Là encore, l'intention de l'auteur s'exprime à travers tout un faisceau de références, dont la simplicité des unes ne fait que masquer la complexité des autres. C. L. Barber a attiré l'attention sur l'analogie entre les vingt-quatre ans du pacte, les vingt-quatre heures de la journée et la fiction entretenue par l'auteur, selon laquelle l'œuvre aurait été achevée à minuit, comme la vie de Faust, et peut-être comme sa représentation théâtrale. ${ }^{13}$ En effet, la phrase "Terminat hora diem : terminat Author opus ", précède le traditionnel FINIS. Barber interprète cela comme un moyen, pour l'auteur, de se dissocier de son personnage, dont il ferait une sorte de bouc émissaire. Allons plus loin, et rappelons, après d'autres, que les dernières minutes de Faust correspondent à un nombre égal de vers dans le dernier monologue de l'acteur qui joue le rôle. ${ }^{14}$ Or, il se trouve aussi que le texte de 1616 comporte exactement vingt-quatre parties: vingt scènes dramatiques ${ }^{15}$ et quatre interventions du choeur. Toutes ces correspondances appellent des questions sur les rapports entre l'auteur et son ouvre, le créateur, avec ou sans majuscule, et sa créature. Barber pense que Marlowe se veut bourreau de son personnage, executioner, théorie intéressante si l'on se souvient que Wagner nomme la salle à manger où se scelle l'association de Faust et de ses " parrains "Valdes et Cornélius, " the place of execution» (ii, 19). Je crois que nous sommes invités à rapprocher les rapports de pouvoir qui existent entre le dramaturge et son personnage, entre Dieu et Faust. Une analyse sémantique aura tôt fait de le confirmer. C'est d'abord le prologue qui érige le public en tribunal, à la fois du jugement artistique et du jugement dernier : succession volontaire de perform et de "the form of Faustus' fortunes, good or bad " (7-8). Le parallélisme se poursuit insidieusement avec des mots 
comme plot (dans son triple sens de jardin, intrigue de théâtre, complot), conjure et conspire, qui finissent par établir un rapport triangulaire entre Dieu, le dramaturge, la créature. Le maître d'œuvre de l'intrigue fait dire à son personnage, Faust : "This night I'll conjure though I die therefor. " (i, 165), ce qui amènera l'expression "conjuror laureate" (iii, 34), elle-même évocatrice du poet laureate, avec l'image "burned is Apollo's laurel bough" (Epilogue, 2). Le troisième côté du triangle apparaît dès le prologue où la chute du nouvel Icare est présentée comme le fruit d'une conspiration céleste : "[...] heavens conspir'd his overthrow " (Prologue, 22). Par ailleurs, le prologue entretient une sournoise ambiguité autour du mot grace, répété dans deux vers successifs en relation avec le mot plot :

So much he profits in divinity,

The fruitful plot of scholarism grac'd,

That shortly he was grac'd with doctor's name (16-17).

Cet emploi insistant et rapproché, éclairé par le rapport entre un dieu comploteur et un dramaturge bourreau intriguant contre son personnage, ne suggère-t-il pas que Faust a toutes les grâces, sauf la grâce, ce don que Dieu distribue "selon son bon plaisir" (IRC, III, xxi, 1, p. 406) ? Pour l'homme Faust, comme pour le personnage de théâtre, pas de franc-arbitre, l'un et l'autre se trouvent embarqués sur les rails d'un temps dont la destination leur échappe. C'est pourquoi il ne faut pas voir dans les vingt-quatre années du pacte une durée réelle et signifiante à l'échelle humaine. Ce nombre nous renvoie à des données étrangères à notre vécu quotidien : à l'union mythique du ciel et de la terre (les douze signes du zodiaque et les douze mois de l'année) ou à la symbolique de l'Apocalypse (les vingtquatre vieillards faisant cercle autour du trône de Dieu comme les heures sur un cadran). Ni le mythe de la naissance du temps, ni la prophétie biblique de la fin des temps ne sauraient constituer la trame de nos jours. Cependant, notre expérience du mouvement pendulaire $d u$ temps nous montre que vingt-quatre représente la somme de mouvements égaux en valeur absolue, mais inverses en valeur algébrique. Somme de plus douze et moins douze, c'est le tout et le rien. Nous pouvons dire que ce nombre situe le pacte dans 
l'atemporalité, ne suggère pas un temps, mais la croisée des chemins du temps (minuit, Noël le 24 décembre et la Saint Jean le 24 juin), cet espace impalpable entre le temps qui n'est plus et celui qui n'est pas encore, et à ce titre adéquatement investi par les esprits médiateurs entre ce monde et l'autre.

Nous touchons là au cœur du drame faustien, et au problème majeur de l'unité d'une œuvre dont le héros, constamment tiraillé entre les tendances adverses d'un dogme qui a subi une mutation traumatisante pour ses fidèles, pris entre le Bon Ange et le Mauvais Ange, entre ses doutes et son désespoir, finira littéralement écartelé. En faisant de la prédestination l'idée clé du salut, le protestantisme a bouleversé les données relatives au temps des hommes, car il n'y a rien qu'ils puissent faire pour se sauver si Dieu ne les a élus. Alors que les "abominables superstitions » romaines mettaient au service du chrétien le plus dévoyé tout un dispositif de salut: les anges, la Vierge, les saints, les reliques, le purgatoire - douloureux, mais purificateur - les messes, les indulgences... la religion réformée a balayé tout cela, à l'exclusion des anges, que Calvin appelle «la gendarmerie du ciel" (IRC, I, xiv, 7, p. 192). Très informé de la nouvelle doctrine, Marlowe sait que le "retour à Dieu ", ${ }^{16}$ défini aussi comme "rénovation" (III, iii, 6, p. 71), "régénération " (multiples exemples), et "réparation" (III, iii, 9, p. 79, et xvii, 5, p. 284), passe par le repentir, que celui-ci n'est pas donné à tout le monde et qu'il y faut encore "l'efficace du Saint Esprit » (III, iii, 21, p. 90). Il ne fait pas de doute que le docteur Faust appartient à la triste catégorie des réprouvés pour lesquels nul retour n'est possible, car Dieu leur " endurcit le cœur et les aveugle" (III, xx, 46, p. 398). La tragédie reflète bien les tensions idéologiques de son époque, par exemple dans la scène vi, où le Bon Ange défend la tradition indulgente de l'Église qui promet à tout péché miséricorde, tandis que Faust s'oppose au Mauvais Ange en plaidant pour la conception la plus magnanime jamais avancée en ses rangs. Il s'agit de la thèse d'Origène, refoulée comme hérétique dès 534, et qui affirme la possibilité de la régénération universelle, même pour les démons $:^{17}$

Be I a devil, yet God may pity me (vi, 15) 
et il ajoute, reprenant la formule du Bon ange :

Yea, God will pity me if I repent (vi, 16).

Le Mauvais Ange représente avec exactitude la position calviniste, car il ne contredit pas Faust, sauf sur sa première affirmation, mais il dit avec assurance: "Ay, but Faustus never shall repent" (vi, 17). Réplique calquée sur l'IRC: "[...] l'apôtre ne dit pas que Dieu leur déniera pardon s'ils [les reprouvés] se convertissent à lui, mais il dit que jamais ne se retourneront à repentance " (III, iii, 24, p. 94). ${ }^{18}$ Faust constate bientôt que le Mauvais Ange a raison et confirme l'explication calviniste de l'impossible retour : " My heart is hearden'd, I cannot repent " (vi, 18). On objectera que Faust en appelle à Dieu quelquefois : "O Christ, my saviour..." (vi, 85-86). La logique calviniste ne sera pas prise en défaut: les réprouvés peuvent faire preuve d'un certain degré de foi, mais il leur manque l'efficace du Saint Esprit (II, ii, 11, p. 35 et 12, p. 36-38). Pourquoi Faust ne peut il faire bon usage des avertissements que lui envoie le ciel ? Parce que si «Dieu adresse sa parole aux réprouvés, c'est pour les faire plus sourds, plus aveugles et plus endurcis" (III, xxiv, 12, p. 463). Pourquoi le remède à ses maux semble-t-il par deux fois à sa portée et cependant hors d'atteinte: "a vial full of precious grace" (xviii, 62-3) et "One drop would save my soul..." (xix, 146-7) ? Calvin répondrait avec la même impitoyable logique: "[Dieu] leur donne remède, mais c'est afin qu'ils ne guérissent " (III, xxiv, 13, p. 465).

Dans cette perspective, il ne reste plus au réprouvé qu'à chercher un dérivatif à son désespoir dans le divertissement, qu'à " tuer le temps " en attendant que ce dernier ne le tue. C'est l'impression que peuvent donner les scènes décrites par $F$. Laroque comme "antimasques ", ${ }^{19}$ et que la critique a, dans son ensemble, jugées incompatibles avec le reste de la pièce. Il me semble au contraire qu'elles s'intègrent parfaitement au schéma idéologique de la tragédie, en suggérant, à côté des tensions exprimées par le Bon Ange (le retour conditionnel), et le Mauvais Ange (le retour impossible), une troisième voie, celle d'avant le christianisme (le retour obligé). Cette vision optimiste, nourrie à la source de la littérature classique et des coutumes populaires héritées de l'Antiquité, transparaît dans les éléments " carnavalesques " de $D F$, qui affirment la réversibilité des situations et du temps lui-même. 
Dans la légende allemande ${ }^{20}$ et sa traduction anglaise, ${ }^{21}$ Faust conduit les jeux dionysiaques de carnaval sous les traits de Bacchus. Sans le nommer, Marlowe suggère clairement la parenté de Faust avec Bacchus/Dionysos, le dieu de l'éternelle renaissance des moissons et des fruits, le dieu du retour par excellence. La référence la plus explicite se situe à la scène xvii, lorsque Faust émerveille la duchesse de Vanholt en lui offrant du raisin en plein hiver. La saison a été définie comme morte: " [...] it is January, a dead time of the winter " (16-17), puis comme stérile : "[...] this time of the year, when every tree/ Is barren of his fruit" (24-25). La duchesse, elle, est enceinte, et Faust, en faisant surgir ces raisins, contredit par un autre symbole de fécondité la stérilité hivernale. L'acte magique retourne l'ordre cosmique qui régit le temps des hommes, il met les deux hémisphères cul par dessus tête à la manière des rites carnavalesques. Nous retrouvons l'évocation de Bacchus, plus précisément de sa naissance, dans le monologue à Hélène, scène xviii. Faust se compare à Sémélé, la mortelle maîtresse de Jupiter détruite par l'insoutenable flamboiement $\mathrm{du}$ dieu, alors qu'elle est enceinte de Bacchus. Jupiter sauve l'enfant en lui faisant terminer sa gestation dans sa cuisse: Bacchus, Sémélé morte et donnant la vie, le père devenu "mère", autant de motifs qui illustrent la réversibilité des situations.

Nous rencontrons le même thème dans les scènes romaines, dont il serait trop long de démontrer la teneur carnavalesque, avec la satire du rituel d'excommunication :

Bell, book and candle ; candle, book, and bell ;

Forward and backward, to curse Faustus to hell (ix, 98-9),

tandis que le prodigieux retour de Bruno à la cour impériale constitue un véritable pied de nez à l'autorité papale et à ses pompeuses certitudes. Les démélés grotesques de Faust et Benvolio appartiennent à la même veine. Benvolio, encore sous l'emprise de Bacchus pour avoir trop bien fêté l'arrivée de Bruno, fait un pari stupide :

B. :[...] And thou bring Alexander/and his paramour before the emperor, I'll be Acteon and/turn myself to a stag.

F. :And I'll play Diana and send you the horns presently. (xii, 50-53). 
Il est important de noter que Benvolio a refusé de se joindre à la compagnie, et observe la scène de sa fenêtre, encore en bonnet de nuit, et qu'il ne tarde pas à se rendormir. C'est alors que l'on remarque : "Two spreading horns most strangely fastened/Upon the head of young Benvolio" (73-4), et que le duc de Saxe se demande: "What, is he asleep, or dead ?" (75). L'empereur, qui a déjà défini la libération de Bruno comme une rédemption (xii, 9), intercède auprès de Faust en employant un terme directement en rapport avec la tragédie :

Let me entreat you to remove his horns ; He has done penance now sufficiently (105-6)

Si bien que les cornes deviennent le signe amovible du rabaissement qui débouche sur le renouveau. Cette scène, où Faust se fait traiter de " cuckold-maker", ressemble à la transposition d'un mythe celtique de renouveau dont Merlin est le héros. ${ }^{22}$ Apprenant dans les astres que son épouse le trompe, il se rend au palais, monté sur un cerf et suivi des animaux sauvages de la forêt. Les deux amants l'observent de la fenêtre et se moquent de lui, Merlin se venge de son rival en lui jetant à la tête les cornes du cerf, animal chtonien lié au renouveau de l'âme du monde. Cocufieur cocufié, le malheureux amant de la dame doit céder la place et descendre au royaume souterrain. Le rapport entre le cerf et le changement périodique du temps appartient à une antique tradition déjà attestée chez Aristote. Devenu vieux, le cerf attire le serpent hors de son trou par son haleine, et l'avale. Habité par le feu du serpent, il devient l'autre, en même temps que l'altéré : sa peau tombe et il retrouve sa jeunesse pour aussi longtemps qu'il s'abstiendra de se désalterer. ${ }^{23}$ Le Bestiaire médiéval a christianisé la légende en faisant du cerf un symbole du Christ qui, en tuant le serpent, c'est-à-dire le diable, apporte le renouveau à l'humanité, et voici ce qu'il dit :

Before his cure he casts his horns

In wood or in a brake of thorns :

And that is how this wild deer

Renews his youth : you learn it here. 


\section{Suit le traditionnel Explicatio :}

There must we drink God's word

Till all our sins are cured.

There shall we cast aside,

As stag his horns, our pride,

In sight of God our youth restore,

And guard our virtue evermore. ${ }^{24}$

Sans doute était-il urgent de christianiser, car le dieu cornu des Celtes avait laissé une empreinte si profonde dans les mentalités que rien n'avait pu empêcher les populations de se déguiser en cerf ou en boeuf pour appeler le retour du temps fertile durant les calendes de janvier. ${ }^{25}$ Ayant accepté de débarrasser de ses cornes l'insolent Benvolio, Faust poursuit la démonstration de la réversibilité des situations avec la scène de pseudo-décapitation. Il y joue à la fois le rôle de la victime et du médecin qui, dans les "Mummers' plays " effectue la cure miraculeuse; d'ailleurs, il a comme lui, beaucoup voyagé. ${ }^{26}$ Benvolio passe de la jubilation: "The devil's dead; the furies now may laugh " (xiii, 45), à l'indignation : "Zounds, the devil's alive again" (67). Au delà de sa spontanéité inconsciente, ce juron doit réveiller dans nos mémoires l'écho du postulat origéniste de Faust à la scène v: "Be I a devil, yet God may pity me ", car il dit exactement que par les blessures du Christ le diable a retrouvé la vie. Dans le temps qui sépare les deux exclamations de Benvolio, chaque partie de la tête tranchée a reçu, verbalement, une nouvelle affectation grotesque qui la défait et la recrée (55-64), sur le mode des jeux populaires de la période hivernale. Citons par exemple ce qui arrive au bélier de Derby, dans la chanson du même nom autrefois associée au sacrifice rituel de l'animal dans les Midlands du nord :

All the women in Derby came begging for his hide

To make some leather aprons to last them all their lives [...]

All the lads in Derby came begging for his eyes

To kick them up and down the street for footballs and bulls eyes [...]

All the ringers in Derby came begging for his tail

To ring the Derby passing-bell that hangs up on the wall. ${ }^{27}$ 
Objet de démonstration du retournement des situations en apparence les plus irréversibles, la tête dé- et recomposée anticipe le corps remis bout à bout de la "victime ", et le retour des cornes sur la tête de Benvolio (xiv, 11-13). Comme le rival de Merlin, il ira momentanément cacher sa disgrâce dans les obscures profondeurs d'un château qui jouxte la forêt, forme de substitution du monde souterrain :

I have a castle joining near these woods, And thither we'll repair and live obscure Till time shall alter these our brutish shapes (xiv, 21-3).

Si l'on se souvient que Calvin nomme le repentir "réparation ", on appréciera le jeu de mots sur "repair " associé à cette confiance dans les capacités régénératrices du temps.

L'unité thématique de la pièce ne serait-elle pas justement cette révolte de la conscience face à un dogme qui fait du chemin de la damnation une voie à sens unique, alors que depuis la nuit des temps l'homme porte au plus profond de soi la certitude de l'éternel retour du renouveau, de la reverdie obligée des prairies et de l'âme du monde ? Bien sûr Faust descend au monde souterrain symbolisé par la gueule d'enfer, pour y être damné perpétuellement, mais toute gueule ingère, digère et restitue ce qu'elle absorbe sous une forme fécondante. De plus, l'image finale du corps démembré à la manière de ce que les titans firent subir à Dionysos, appelle la question du retour. Question sans réponse, certes, mais Marlowe a déjà comparé Faust à Aréthuse (xviii, 116-7), la nymphe changée en rivière souterraine qui resurgit à Délos. ${ }^{28}$ Symbole courant des réapparitions en tout genre, c'est, ironiquement, celui que choisira Thomas Browne pour décrire le retour cyclique des hérésies :

For indeed, heresies perish not with their authors, but, like the river Arethusa, though they lose their currents in one place, they rise up again in another. ${ }^{29}$ 


\section{NOTES}

1) Ed. J. D. Jump, The Revels Plays, a University Paperback Text (London : 1968).

2) E. R. Leach, Critique de l'Antropologie (Paris : P.U.F., 1968), chap. VI. Mircea Eliade, Le Mythe de l'Éternel Retour (Paris : Collection Idées, 1969).

3) Analogie frappante, en anglais, entre tomb et womb, grave et la femina gravida, cf, Romeo and Juliet, II, iii, 9-10:

The earth, that's nature's mother, is her tomb

What is her burying grave, that is her womb,

et Donne, "The Anatomy of the World", vers 451-4:

For though the soul of man

Be got when man is made 'tis born but then

When man doth die. Our body's as the womb

And as a midwife death directs it home.

4) Euvres, trad., G. Combès (Paris: Desclée de Brouwer, 1960), vol.37, XXII, 1, p. 525.

5) Importance des mots commence et end dans le premier monologue, voir E. A. Snow, "Marlowe's Doctor Faustus and the ends of desire", Selected Papers from the English Institute (1977), pp. 77-9 notamment.

6) Stephen Greenblatt, "Marlowe and Renaissance Self-Fashioning ", dans Two Renaissance Myth-Makers, Christopher Marlowe and Ben Jonson, Selected Papers from the English Institute, n. s. 1 (1975-6), pp. 41-69.

7) C'est l'homologue de Mercure dans la littérature hermétique, Agrippa l'appelle Ophiel tout court; voir Matila Ghyka, Philosophie et Mystique du Nombre (Paris : 1971), note 1, p. 89.

8) Les connotations érotiques de come suggèrent l'identification de l'âme et du sexe source de maint calembour au Moyen-Age, surtout dans la satire de la corruption du clergé. L'iconographie du diable se plaît aussi à inverser le matériel et le spirituel, le démon a souvent un phallus planté au milieu du visage, et une langue à la place du sexe.

9) "Marlowe's Doctor Faustus as an Inverted Saint's Life", Studies in Philology, 63, (1966), pp. 565-77.

10) Institution de la Religion Chrétienne, éd., J. D. Benoit, 5 volumes (Paris : Librairie Jean Vrin, 1959-63), IV, xv, 1, p. 318. J'ai modernisé l'orthographe, toute référence ultérieure à cette œuvre sera indiquée par l'abréviation IRC.

11) Pauline Honderich, "John Calvin and Doctor Faustus ", Modern Language Review, 68 (1973), p. 3

12) C. L. Barber, "The Form of Faustus'Fortunes Good or Bad ", Tulane Drama Review (1964), ne voit, p. 108, dans la scène du pacte, qu'un rituel inversé par rapport à celui de la communion.

13) Article cité, p. 118.

14) Harry Levin, The Overreacher (Harvard University Press, 1952), pp. 127-8. H. Politzer, "Of Time and Doctor Faustus ", Monatshefte, LI, 4 (1959), p. 147. 
15) Convaincue par l'argumentation de Roy T. Eriksen, je pense que Jump a probablement tort de croire qu'il manque une scène entre $\mathrm{v}$ et vi, et que vii doit seulement être avancée de deux crans.

Voir Jump, édition citée, p. 34, et Eriksen, "The Misplaced Clownage Scene in The Tragedie of Doctor Faustus (1616) and its Implications for the Play's Total Structure ", English Studies, 62, 3 (1981), pp. 249-58.

16) Calvin, "Sermon $n^{\circ} 4$ sur la Passion " dans Euvres de Jean Calvin (Genève : Labor, 1936), vol. III, p. 143.

17) Voir par exemple Jean Danièlou, Origène (Paris : 1948).

18) Cela contredit à la fois Robert $\mathrm{H}$. West et Michael Hattaway qui pensent que le repentir peut à tout moment annuler le pacte. Voir West, "The Impatient Magic of Doctor Faustus ", English Literary Renaissance, 4 (1974), p. 226, et Hattaway, " The Theology of Marlowe's Doctor Faustus ", Renaissance Drama, (1970), p. 74.

19) "En Marge de l'Idéologie: Antimasque et Grotesque dans le Dr Faustus et La Tempête, dans Théâtre et Idéologie: Marlowe, Shakespeare, Actes du Congrès de la Société Française Shakespeare, (Paris : Jean Touzot, 1982), pp. 99-114.

20) Joël Lefèvre, trad., L'Histoire du Dr. Faust (Lyon, Paris : Les Belles Lettres, 1970), chap. 45 à 49 .

21) H. Logeman, éd., The English Faust-Book with Introduction and Notes by H. Logeman (Gand, Amsterdam : 1900), chap. 41 à 45.

22) Episode de la Vita Merlini de Geoffrey de Monmouth, cité dans Claude Gaignebet, Le Carnaval (Paris : Payot, 1974), p. 136.

23) Gaignebet, ouvrage cité, note 1, p. 79. Je n'ai pas trouvé cette légende dans Aristote, néanmoins de nombreux auteurs associent le cerf, le serpent et le renouveau : Pline, Hist-Nat., VIII (L), XI (XLV), Elien, De Natura Animalium, II, 9, Isidore de Séville, Etym., XII, 1, 18 etc.

24) Brian Stone, trad., Medieval English Verse (London: Penguin Books, 6th ed., 1973), pp. 91-2. La source de ce Bestiaire moyen-anglais semble être un texte en vers latin $\mathrm{du} X \mathrm{XI}^{\mathrm{e}}$ siècle.

25) Cela est prouvé par les pénitenciels, voir par exemple, J. T. MacNeil \& H. M. Gamer, éd., Medieval Handbooks of Penance, Columbia University Records of Civil Sources and Studies, XXIX (New York : 1938), p. 420.

En Allemagne, le lundi précédant mardi gras était appelé Hirschmontag, le lundi du cerf, et dans les comédies jouées pour le carnaval le présentateur portait un masque de cerf. Voir Maximilian J. Rudwin, The Origin of the German Carnival Comedy (New York : 1920), p. 34

26) Nombreux exemples dans Alex Helm, The English Mummers' Play, Mistletoe Series n 14 (Woodbridge, Suffolk : 1981).

27) Thomas Pettitt, "Folk-Play in Dr Faustus ", Folklore (1980) (1), p. 74.

28) Ovide, Les Métamorphoses, trad. J. Chamonard (Paris: Garnier Flammarion, 1966), V, pp. 152-3.

29) Religio Medici, éd. R. H. A. Robbins (Oxford: Clarendon Press, 1972), sect. 6, p. 7. 